\title{
3D printing of bone scaffolds with hybrid biomaterials
}

\author{
a*Bankole. I. Oladapo, ${ }^{\text {aS }}$. Abolfazl Zahedi, ${ }^{\text {b A.O.M. Adeoye }}$ \\ $a^{*}$ School of Engineering and Sustainable Development, De Montfort University, Leicester, UK \\ ${ }^{b}$ Mechanical and Mechatronics Department, Afe babalola University, Ado Ekiti, Nigeria \\ *Corresponding author, Tel: +44(0)116 201 3971, E-mail: P17243433@my365.dmu.ac.uk
}

\begin{abstract}
In this research, a novel hybrid material bone implant manufacturing through the integration of two materials using additive manufacturing (AM) technology is proposed. Biomimetic application can manufacture high strength biomechanical implants with optimised geometry and mass. The combination of polymers allows a significant leap in the development and production of a great diversity of components and applications of biomaterials. A novel hybrid scaffold with a poly lactic acid (PLA) matrix reinforced with carbohydrate particles (cHA) is analysed using digital surface software in the mass proportions of 100/0, 95/5, 90/10 and 80/20 for application in tissue and regenerative engineering, seeking a higher proposition strength of PLA. Filaments are used to fabricate scaffolds by 3D printing, using the fused deposition method. The frameworks are submitted to bioactivity tests, surface roughness evaluation, apparent porosity and mechanical analysis. Analysis of the microstructure of the composite particle evaluates the 3D surface luminance structure and the profile structure. Cross-sectional views of the specimens are extracted and analysed, and the surface roughness, waviness profile, and Gaussian filter of the structures are observed. In summary the structures are checked and analysed by SEM and EDS where possible, to observe the bioactive behaviour of the materials. The relationship between cHA content and roughness is shown to be proportional. The mechanical properties are shown to be affected by the reduced interaction between the PLA matrix and the cHA particles.
\end{abstract}

Keywords: tissue engineering; bone generation; PLA/cHA; 3D printing; hybrid manufacture

\section{Introduction}

Findings from population statistical research institutes point to a rising life expectancy in the world population. As humans age, the risk of compromising organs, tissues and the body as a whole increases. Tissue engineering seeks to use biomaterials to replace conventional transplants. A biomaterial is defined as any substance or combination of materials, other than drugs or synthetic natural materials, which can be used, for any period, to partially substitute for any tissue, organ or function of the body for the purpose of maintaining or improving the lifespan of an individual [1]. A fundamental requirement of a biomaterial used in clinical medicine is that it is biocompatible, that is, it can coexist with human tissue without causing any undesirable or inappropriate effects $[2,3]$. Biomaterials can be classified into three main categories: bioinert, intolerant materials that are not capable of inducing any biological interfacial bond between the implant and the host tissue [4, 5]; bioactive, capable of interacting with body tissues, forming chemical or biological bonds and favouring the development of processes such as implant fixation, colonisation and tissue regeneration; and bioreabsorbable, materials that are gradually reabsorbed until they disappear entirely and are wholly replaced by new tissue in vivo. In general, biomaterials can be classified into: natural, 
from plants and animals; and synthetic, which can be sub-categorised into metals, synthetic polymers, ceramics and composites [6,7].

Metal is a common materials used in the biomedical field for implants. Its high tensile strength and fatigue characteristics make it suitable for a variety of applications, such as dental implants, and joints such as knees and hips [8, 9]. However, metals have limitations related to corrosion, which can lead to toxicity or hypersensitivity reactions. Polymers are also widely used thanks to their full range of compositions, properties and shapes, in the form of matrixes, fibres, films and gels [10, 11]. Their processing possibilities enable the fabrication of complex forms and structures. If the final goal is to manufacture small components with complex geometry, understanding mechanical properties at a micro-scale is paramount $[12,13]$. Traditional ceramics have high compressive strength but low tensile strength. Most ceramic materials are characterised by being hard and brittle, with a modulus of elasticity typically higher than that of bone. These characteristics stimulate the study of this class of material as a bone substitute, due to its easy confirmation and mechanical properties. Composite material is formed by the joining of elements, resulting in a new material with better properties $[14,15]$. Composites have been increasingly studied over the years, because they have a high potential to generate combinations of features that cannot be obtained with conventional materials. This group of materials has recently come into use in the field of biomaterials, but has the possibility of being mechanically and biologically similar to human tissue.

A particular classification of composites is organic-inorganic hybrid materials. These materials are formed by the combination of two or more organic and inorganic phases. In general, they are produced when there is a need for properties not found in materials with a single constituent [16]. They can have better mechanical, optical and thermal properties, combining the thermal and chemical stability of ceramic materials with the processability and flexibility of organic compounds and polymers. Advances in the development of biomaterials have been shown to be an essential tool for tissue engineering [17]. These materials can promote regenerative processes through the active transport of cellular populations and therapeutic agents, as well as providing structural support with similar mechanical properties to tissue [18]. Biomaterials should ideally degrade at a rate comparable to the growth of new tissues at the site of implantation. Synthetic biomaterials, such as calcium phosphate ceramics, hydroxyapatite (HAp) and carbonatite hydroxyapatite (cHA), are commonly used as osteoconductive bone graft materials. Carbonatite has carbonate groups replacing phosphate groups, but these materials, when used alone, have low tensile strength and a high susceptibility to fractures. Therefore, these materials generally serve as reinforcing filler for elements with a more elastic nature, such as PLA, which is a biodegradable and biocompatible polymer, widely used in biomedical and food engineering. The aim of this research is the production of poly (lactic acid) hybrids containing $\mathrm{cHA}$, in various mass/mass proportions, by fused decomposition method (FDM). The purpose of the output frameworks is application in bone regeneration. The evaluation of bioactivity in the structures of pure PLA and various ratios of cHA and their mechanical behaviour is undertaken through compression tests. AccuCT microCT analysis software is used for automatic bone segmentation, in order to calculate morphometry parameters, including trabecular and cortical volumes, and calibrate the bone mineral density analysis. 


\section{Materials}

\subsection{Biomaterials for bone regeneration}

Biomaterials are widely used in tissue engineering for the regeneration of bone tissues. In this research, we present the results of a study of the biochemical properties of biomaterials and their role in cell growth. The focus of this work is the application of scaffolds made of a composite material, composed of poly (lactic acid) matrix reinforced with cHA particles. PLA is an aliphatic, thermoplastic, semi-crystalline or amorphous, biocompatible, biodegradable polyester, synthesised from lactic acid obtained from renewable sources [19, 20]. Using FDM, 3D complex architectural scaffolds with various pore sizes and porosities can be generated by computer-controlled design, layer-by-layer printing and a solidifying process, as shown in Figure 1. The process involves the formation of bone with PLA/cHA with a fused deposition scaffold produced by 3D printing. Optical images illustrate the 3D periodic structure with a simple tetragonal symmetry showing integrity interfaces formed in layers and a radial structure comprised of alternating layers deposited in concentric fill patterns [21]. Figure 1 also shows an SEM section of 3D deposited scaffolds with homogeneous $1 \mathrm{~mm}$ fibre spacing, showing typical fibre diameters and pore geometries at magnification of 20 .
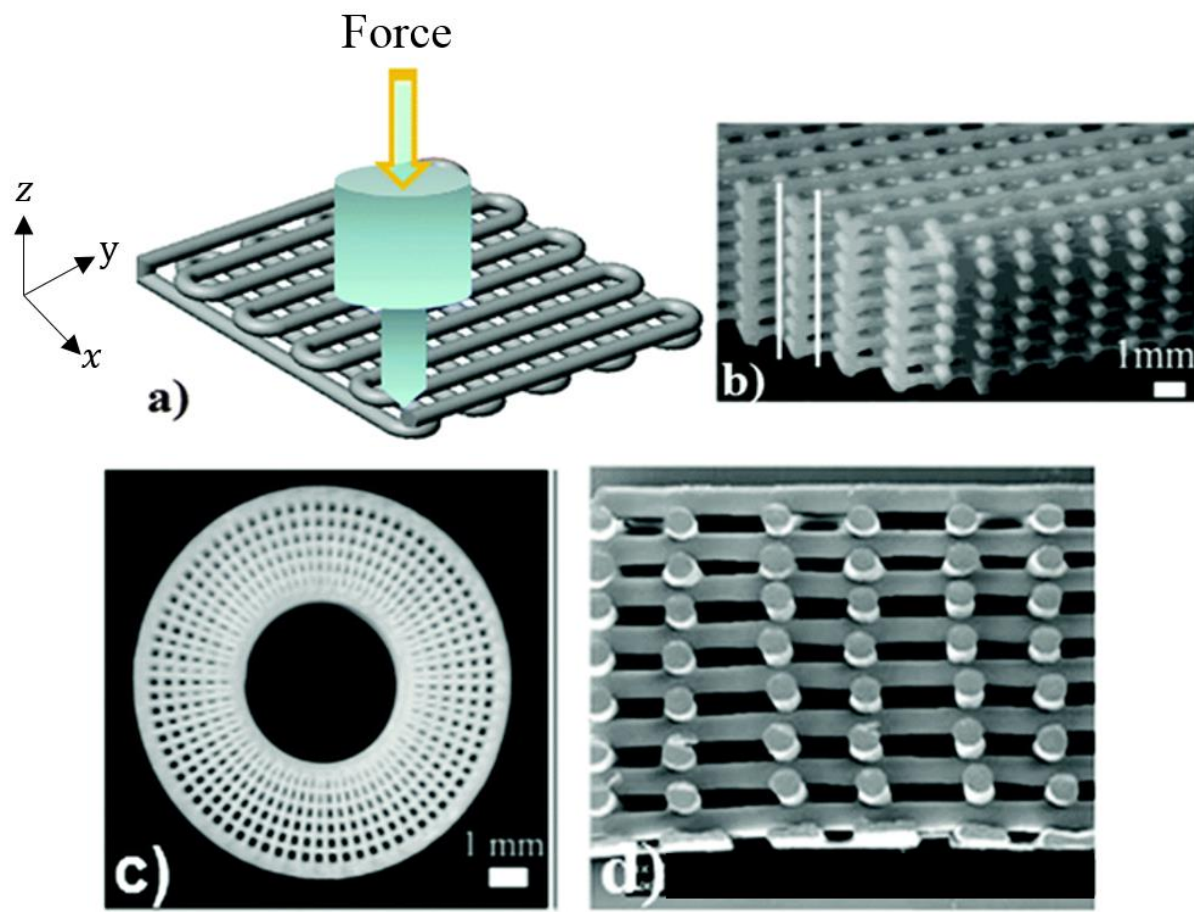

Figure 1. The process of formation of bone with PLA/cHA by fused deposition, (a) two layer scaffold produced by 3D printing: optical images illustrating, (b) 3D periodic structure with a simple tetragonal symmetry showing integrity interfaces formed in layers, (c) 3D radial structure comprised of alternating layers deposited in concentric fill patterns, (d) SEM section of 3D deposited scaffolds with uniform $1 \mathrm{~mm}$ fibre spacing showing average fibre diameters and pore geometries at magnification of 20

Poly (lactic acid) is a polymer produced by the polycondensation reaction or lactide ring opening. The fundamental constitutional unit of PLA is lactic acid. Lactic acid is an $\alpha$ hydroxy acid with an asymmetric carbon atom, with two optical isomers: L-lactic acid (PLLA) and D-lactic acid (PDLA). The L-isomer is produced in humans and other mammals, while the D and L enantiomers are produced in bacterial systems [21]. Lactic acid can be 
derived from renewable resources, such as ethanol or acetaldehyde, or from coal or oil. However, the vast majority of lactic acid produced today is obtained by bacterial fermentation of simple sugars. Table 1 shows the main properties of PLA and its polymer, being amorphous without a melting point as described by the manufacturer. $T_{g}$ is glass transition temperature and $T_{m}$ is melting temperature.

Table 1. Mechanical and thermal properties of PLA

\begin{tabular}{lccc}
\hline Property & \multicolumn{3}{c}{ Type of PLA biopolymer } \\
\hline & PLA & PLLA (Natureworks ${ }^{\text {TM }}$ 4032D) & PDLA (Natureworks ${ }^{\text {TM }}$ 4031D) \\
Density $\left(\mathbf{g} / \mathbf{c m}^{\mathbf{3}}\right)$ & 1.24 & 1.30 & 1.27 \\
Stress $(\mathbf{M P a})$ & 41 & 40 & 40 \\
Modulus of elasticity (GPa) & 1.6 & 2.7 & 3.0 \\
Poisson ratio (\%) & 2.5 & 3.1 & 2.1 \\
$\boldsymbol{T}_{\boldsymbol{g}}\left({ }^{\mathbf{0}} \mathbf{C}\right)$ & 55.3 & 55 & 50 \\
$\boldsymbol{T}_{\boldsymbol{m}}\left({ }^{\mathbf{0}} \mathbf{C}\right)$ & 151.5 & 167.9 & - \\
\hline
\end{tabular}

PLA is a biodegradable polymer, and its main route of degradation is by cleavage of the ester linkages by hydrolysis, leading to a progressive reduction in molecular weight. This degradability, along with its biocompatibility, is the reason PLA has been widely used in the last four decades for medical applications, including suture and tissue engineering frameworks [22]. Hydroxyapatite, $\mathrm{Ca}_{10}\left(\mathrm{PO}_{4}\right)_{6}(\mathrm{OH})_{2}$, also known as HA or HAp, is a natural mineral constituent of bone, representing 30 to $70 \%$ of the mass of bones and teeth and with a $\mathrm{Ca} / \mathrm{P}$ ratio of 1.67 . Synthetic hydroxyapatite has the combined properties of bioactivity and biocompatibility with the human body and can promote bone growth thanks to its excellent biological response. These properties, combined with their high capacity to adsorb and absorb molecules, make hydroxyapatite excellent support for the long-acting anticancer drugs used in the treatment of bone tumours. The structure of hydroxyapatite allows isomorphic cationic and anionic substitutions with great ease. $\mathrm{Ca} 2+$ can be replaced by metals such as $\mathrm{Pb} 2+$, $\mathrm{Cd} 2+, \mathrm{Cu} 2+, \mathrm{Zn} 2+, \mathrm{Sr} 2+, \mathrm{Co} 2+, \mathrm{Fe} 2+$ etc., the phosphate groups by carbonates and vanadates, and hydroxyls by carbonates, fluorine and chlorine [23]. These substitutions may alter crystallinity, network parameters, crystal size, surface texture, stability and solubility of the hydroxyapatite structure. The characteristic of HAp, at the level of its structure, which allows numerous ion or ionic group substitutions, can be changed. Such changes generate a series of perturbations in the crystalline structure, which causes the crystallinity to decrease, while increasing its capacity for reabsorption in a physiological medium. The substitutions that are generally used are carbonate ions, in a concentration of $4-8 \%$ by weight in natural bone [24]. Figure 2 shows an SEM image of the PLA/cHA scaffold framework pores at a various ratios. 


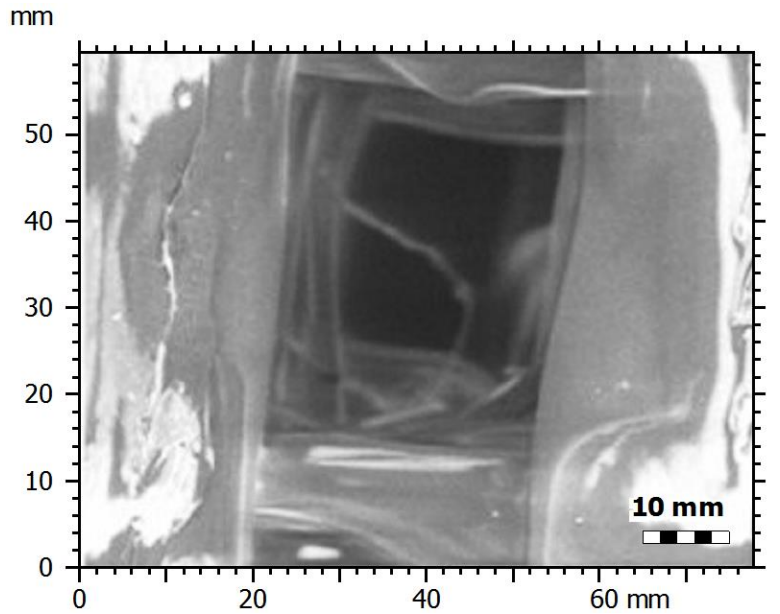

(a)

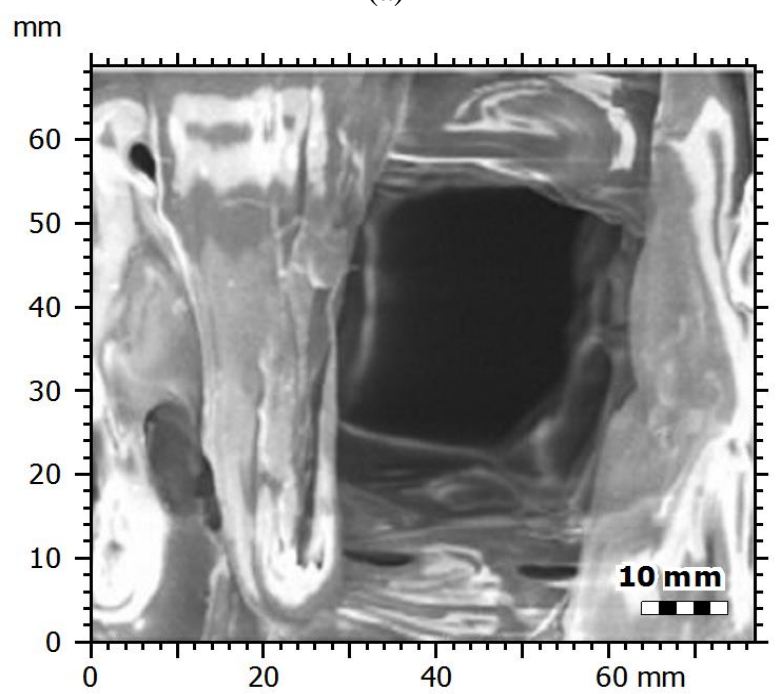

(c)

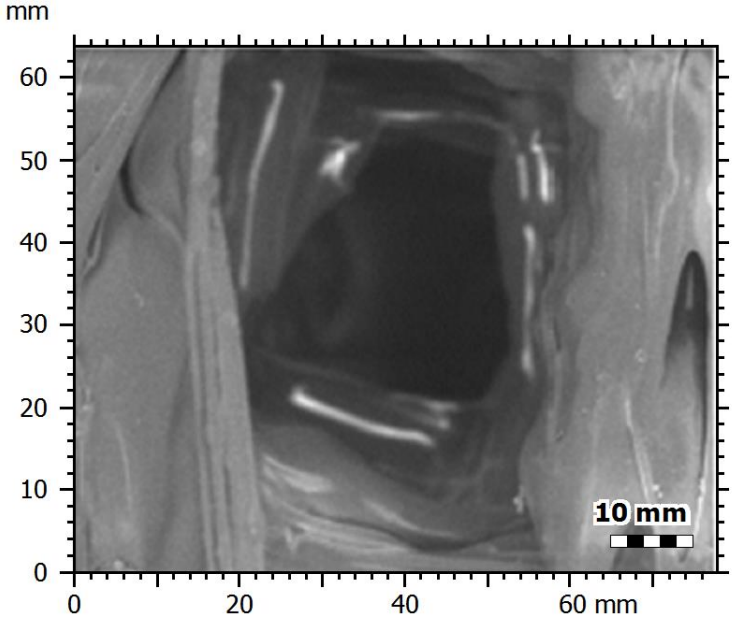

(b)

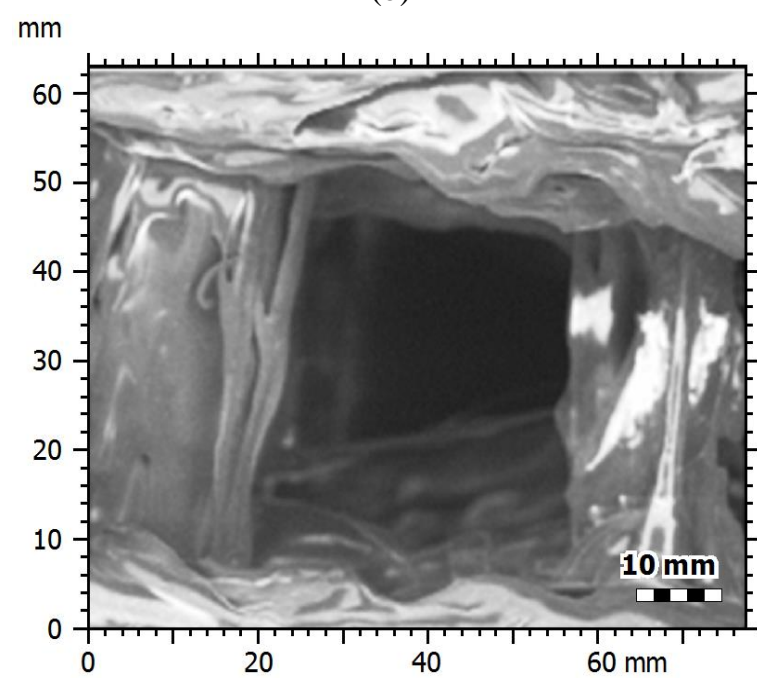

(d)

Figure 2. SEM image of the PLA/cHA scaffold framework pores at (a) $100 / 0$, (b) $95 / 5$, (c) $90 / 10$, and (d) 80/20 ratios

Changing the structure of HAp results in cHA, which has the same physicochemical properties as HAp. It can improve the kinetics of absorption, making it faster, and improve the mechanical properties, making it a biomaterial increasingly similar to natural bone. Different types of cHA can be obtained depending on the site occupied by $(\mathrm{CO} 3)^{2-}$ replacing either $(\mathrm{OH})^{-}$or $(\mathrm{PO} 4)^{3-}$, called type A and type B substitution [25]. Type A is prepared at high temperature $\left(1000{ }^{\circ} \mathrm{C}\right)$, and type $\mathrm{B}$ at lower temperature. The preparation method influences the kind of substitution generated. The two types of replacement have opposite effects on the network parameters. Type A change, in which the largest planar group of $(\mathrm{CO} 3)^{2-}$ replaces the $(\mathrm{OH})^{-}$group, causes expansion in the a-axis and contraction in the dimensions of the c-axis [26]. Meanwhile, substitution of type $\mathrm{B}$, in which a small plane group of $(\mathrm{CO} 3)^{2-}$ replaces the largest tetramer group of (PO4) ${ }^{3-}$, causes a contraction in the aaxis and expansion in the dimensions of the c-axis. In a previous study [27], the presence of type B substitutions is shown to cause a decrease in crystallinity and an increase in solubility both in vitro and in vivo. The surface of A-cHA is shown to have a lower affinity for the human trabecular osteoblastic cell, compared to HA. In another study [25], the compressive strength of porous bodies of cHA is shown to be twice as high as the resistance in analogous 
porous HA samples. Mechanical strength is, therefore, less critical for porous cHA structures. It is also shown that cHA implants have higher osteoconductive and bioabsorption properties than similar HA implants.

\section{Method}

The composite used in this work consists of a matrix of poly (lactic acid) (PLA) with various levels of carbonated hydroxyapatite (cHA). PLA (Ingeo 2003D from NatureWorks) containing 4\% DPLA isomer is common in the market. Carbonated hydroxyapatite is synthesised at $37^{\circ} \mathrm{C}$, to have a particle diameter of less than $90 \mu \mathrm{m}$. Table 2 shows the printer setting for fabricated scaffolds.

Table 2. Printer settings for preparation of dumbbell-shaped specimens.

\begin{tabular}{lcc}
\hline Printer setting & Horizontal build direction & Vertical build direction \\
\hline Nozzle diameter [mm] & 0.4 & 0.4 \\
Perimeter shells & 2 & 2 \\
Bottom solid layers & 2 & 2 \\
Top, solid layers & 2 & 2 \\
Layer height [mm] & 0.15 & 0.15 \\
Infill [\%] & 99.9 & 99.9 \\
Infill pattern & 1 linear & 1 inear \\
Head travel speed [mm/s] & 100 & 120 \\
Print speed [mm/s] & 50 & 70 \\
Nozzle temperature $\left[{ }^{\circ} \mathbf{C}\right]$ & 200 & 195 \\
Platform temperature $\left[{ }^{\circ} \mathbf{C}\right]$ & 65 & 65 \\
\hline
\end{tabular}

To produce a biomaterial with characteristics, such as mechanical strength, fracture toughness and rigidity, similar to human bone tissue, ceramic particles and nanoparticles, nano carbon tubes and HA particles/fibres are incorporated, as in the studies of many other researchers. Table 3 shows the mechanical properties of cortical (compact) and trabecular bones.

Table 3 Mechanical behaviour of compact and trabecular bones after [28]

\begin{tabular}{|l|l|l|l|l|}
\hline \multirow{2}{*}{ Compact bone } & \multicolumn{3}{|c|}{ Strength (MPa) } & \multirow{2}{*}{ Modulus of elasticity (GPa) } \\
\cline { 2 - 4 } & Compression & Tension & Twist & \\
\hline Trabecular bone & $100-230$ & $78-150$ & $50-150$ & $7-30$ \\
\hline
\end{tabular}

\subsection{Bioactivity test and Chauvenet criterion}

The bioactivity was evaluated and reproduced in vitro using the biomimetic method, based on ISO 10993, in which the frameworks were immersed in a solution known as synthetic body fluid (SBF), which mimics body fluid, containing the ions present in blood plasma. When the ions in this solution are supersaturated, it is possible to observe the deposition of a layer on the surface of the scaffold with varying morphology. Triplicates of PLA/cHA frameworks were used in the mass/mass ratios of 100/0, 95/5, 90/10 and 80/20. The samples were immersed in FBS solution and placed in an oven at $37^{\circ} \mathrm{C}$ for 14 and 21 days, then the analysis of the chemical composition of the surface and its morphology were carried out [24]. In order to eliminate the discordant data obtained, further analysis was carried out with digital surface 
software. The Chauvenet criterion was used to determine which values should be excluded. This criterion eliminates a measure if:

$$
d j=\left|\left(y_{j}-y\right)\right|>d_{c h}
$$

where $d_{c h}$ is the Chauvenet rejection limit, defined by:

$$
\int_{-\infty}^{d_{c h}} G(\eta) d \eta+\int_{d_{c h}}^{+\infty} G(\eta) d \eta=\int_{-d_{c h}}^{-d_{c h}} G(\eta) d \eta=\frac{1}{2 n}
$$

where $G(\eta)$ is the Gaussian filter function. In this criterion a measured value can be rejected if the probability $m$ of obtaining the deviation from the mean is less than $\frac{1}{2 n}$, where $n$ is the number of measurements.

\subsection{X-ray dispersive energy spectroscopy (EDS)}

In this analysis, the chemical elements present in the frameworks were identified using Quantax 70 Brucker equipment. A comparison was made between the $\mathrm{Ca}$ and $\mathrm{P}$ peaks present in the scaffolds with various concentrations of cHA. The analysed frameworks were submitted to the bioactivity assay for 0,14 and 21 days in order to verify the variation in the intensity of the calcium and phosphorus peaks. X-ray diffraction was used to evaluate calcium phosphate deposition in 80/20 PLA/cHA frameworks after 21 days of bioactivity assay. PLA/cHA 100/0 and 80/20 frames were not submitted to the test and carbonatites were characterised for comparative purposes. The characterisations were performed using a Shimadzu XDR-6000 diffractometer, operated in a scanning range of 2 degree from 5 degree to 80 degree, in continuous scanning, with a scan speed of 2 degree/min.

\subsection{Mechanical and surface roughness}

Surface roughness is defined as the sum of the third and fifth order shape differences, overlapping and composing a material's profile [29]. In this test, Taylor Hobson's dosimeter was used with a $2 \mu \mathrm{m}$ radius probe. The parameter evaluated was the roughness average, denominated as $R_{a}$ (roughness average), which is a measure of the depth of roughness. $R_{a}$ is defined as the arithmetic mean deviation of the absolute values of the spacing ordinates $\left(y_{i}\right)$ of the roughness profile points about the midline, within the measurement path $(\mu \mathrm{m})[30]$. Scaffolds produced with mass/mass ratios of 100/0, 95/5, 90/10 and 80/20 were analysed. For each composition, three scaffolds were used, and measurements taken of three different filaments, both along the $\mathrm{x}$-axis and the y-axis. Data were taken for a $3 \mathrm{~mm}$ extended surface located in the central region of each thread. To remove the discrepant values of $R_{a}$, the Chauvenet criterion was used.

\section{Results and discussion}

The surface roughness is presented in Figure 3, which shows the profile roughness and waviness profile of the Gaussian filter, with a cut-off of $2.5 \mathrm{~mm}$. The roughness or arithmetic mean deviation $\left(R_{a}\right)$ was analysed and the values presented in the figure are the averages of the values measured by the rugosimeter in the software for each sample group. 


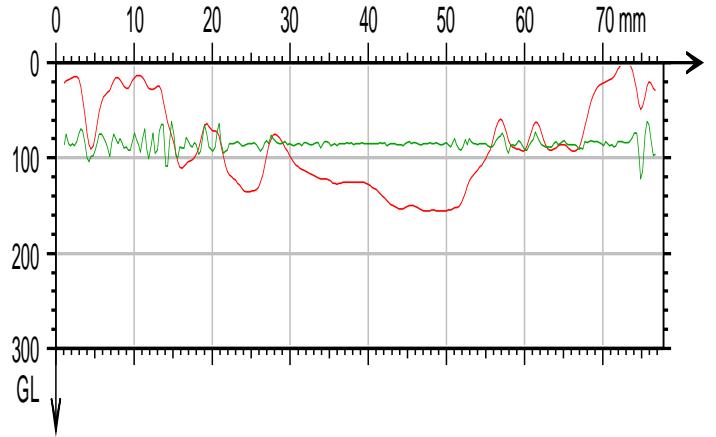

(a)

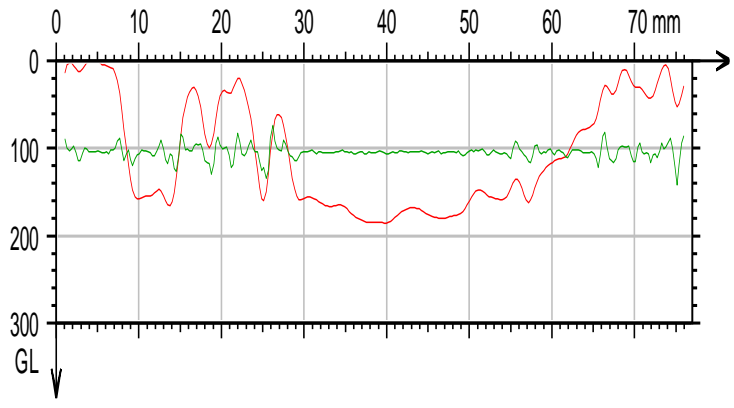

(c)

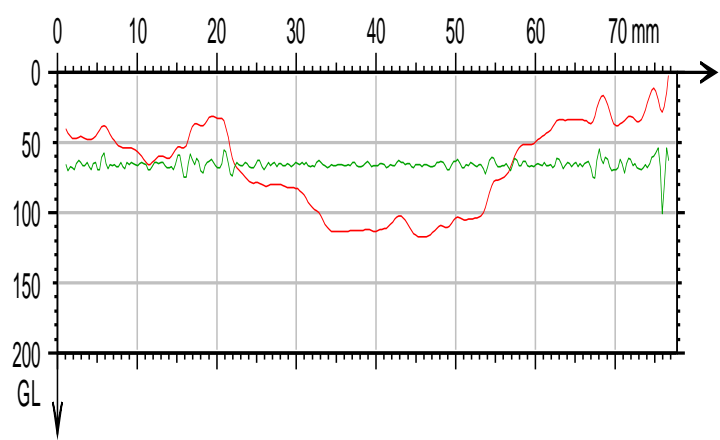

(b)

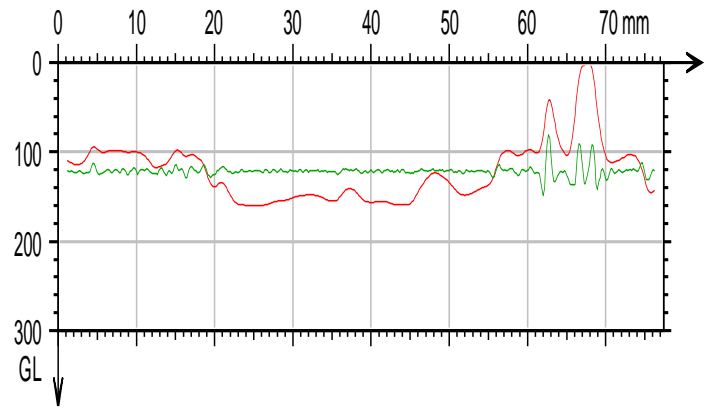

(d)

Figure 3. Average arithmetic roughness and waviness filter extracted from samples PLA/cHA with Gaussian filter $2.5 \mathrm{~mm}$ at (a) $100 / 0$, (b) $95 / 5$, (c) $90 / 10$, and (d) $80 / 20$ ratios

Figure 4 shows an increase in surface roughness as the concentration of carbonatite increases. Considering standard deviation, we can say that the results for roughness lie in the same range of values. The high standard deviation indicates an irregularity between the filaments. Surface roughness is an important factor influencing the biological response to the biomaterial. The increase of this characteristic potentiates the mechanism of mechanical anchoring of particles, improving cell adhesion [29]. The roughness, together with the pores present in the framework, are responsible for mimicking the bone structure, allowing both osteoblast adhesion and local revascularisation. It is possible to observe the graphs with the roughness patterns of the tested frames. The relationship between the percentage of cHA and the roughness was found to be proportional and affected by the poor interaction between the PLA matrix and the cHA particles.

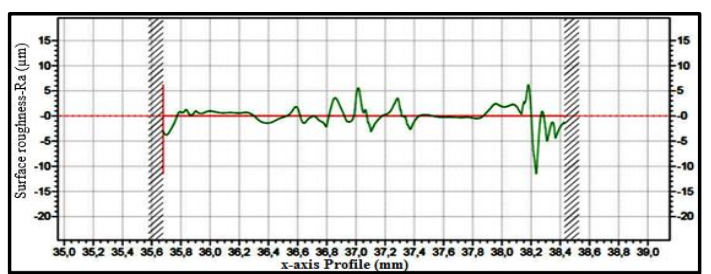

(a)

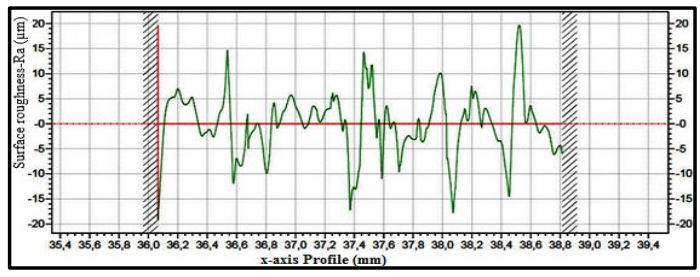

(c)

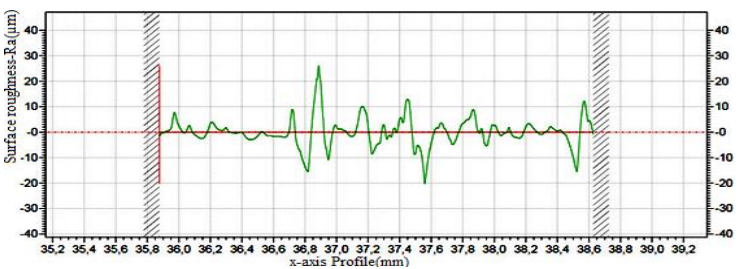

(b)

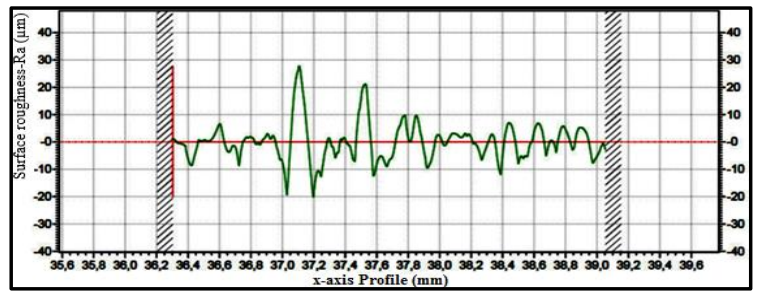

(d) 
Figure 4. Analysis of surface roughness profile along the $x$-axis for (a) $100 / 0$, (b) $95 / 5$, (c) 90/10, and (d) 80/20 samples

Compression tests were carried out on a universal testing machine (Instron 5567) using a 2 $\mathrm{kN}$ load cell at a compression ratio of $1.3 \mathrm{~mm} / \mathrm{min}$. Data was taken every $0.1 \%$ of deformation. For each sample group of PLA/cHA 100/0, 95/5, 90/10 and 80/20, ten samples were used, 5 of which were analysed with the applied load on the XY plane and 5 with the applied load on the XZ plane as shown in Figure 1. The sample sizes were measured with a digital calliper. The assay was performed using $n=5$, and the results for elasticity modulus and mechanical strength are presented as individual values and mean values, accompanied by the respective standard deviation. For a better analysis of these results, the Chauvenet criterion was used to discard distortive values, giving a more reliable average value [31]. The yield of the PLA/cHA composite material obtained was calculated. Each sample group was weighed before and after the process of incorporation of cHA into the PLA matrix. Table 4 shows the values obtained.

Table 4. Partial yield after material mixing

\begin{tabular}{cccc}
\hline Sample & Initial mass $(\mathrm{g})$ & Mass after mixing $(\mathrm{g})$ & Yield (\%) \\
\hline 100/0-PLA/cHA & 120.0 & 106.7 & 88.9 \\
95/5-PLA/cHA & 119.99 & 113.2 & 94.6 \\
90/10-PLA/cHA & 119.91 & 113.11 & 94.17 \\
80/20-PLA/cHA & 120.23 & 110.90 & 92.23 \\
\hline
\end{tabular}

Analysing the returns of the mixtures, we can see the similarity between the yield values for all proportions, with all being observed as close to $90 \%$, indicating a high yield for the technique used.

\subsection{Microstructural bioactivity characterisations}

The analysis of the bioactivity assay was monitored using scanning electron microscopy (SEM) and X-ray dispersive energy spectroscopy (EDS). From the information presented by these characterisations, the proportion of ideal carbohydrate for an excellent bioactive response was obtained in terms of the morphology and the composition of the surface of the scaffolds after the bioactive reaction. Figure 5 shows the spectra obtained by EDS and SEM for the PLA/cHA frameworks in the proportions 100/0, 95/5, 90/10 and 80/20 at the time of the assays. 


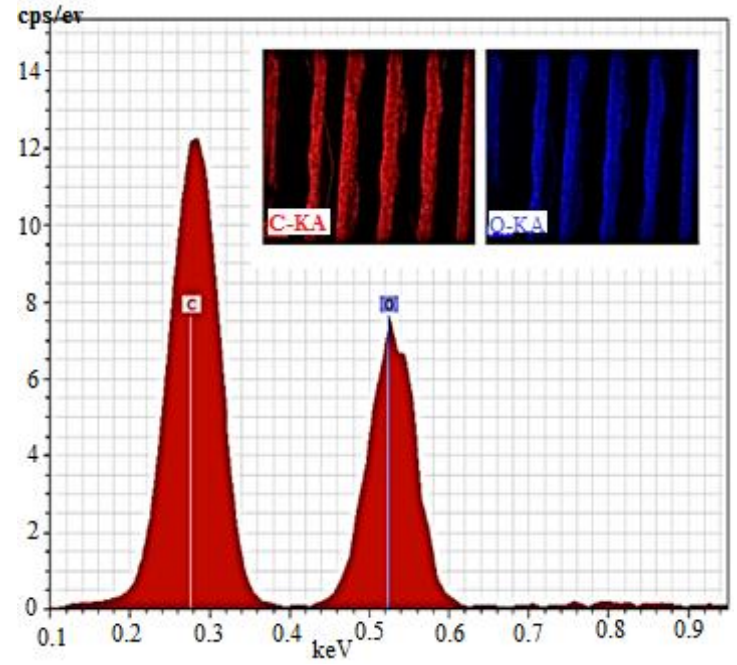

(a)

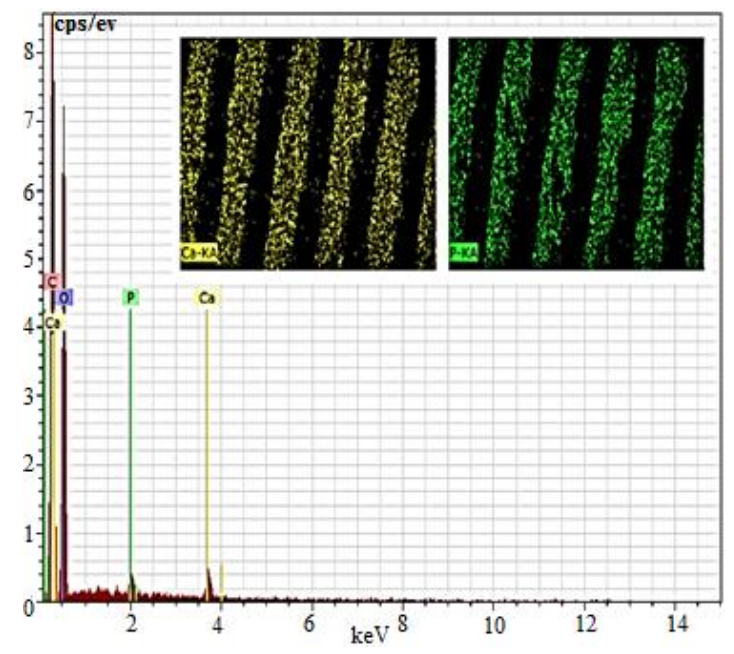

(c)

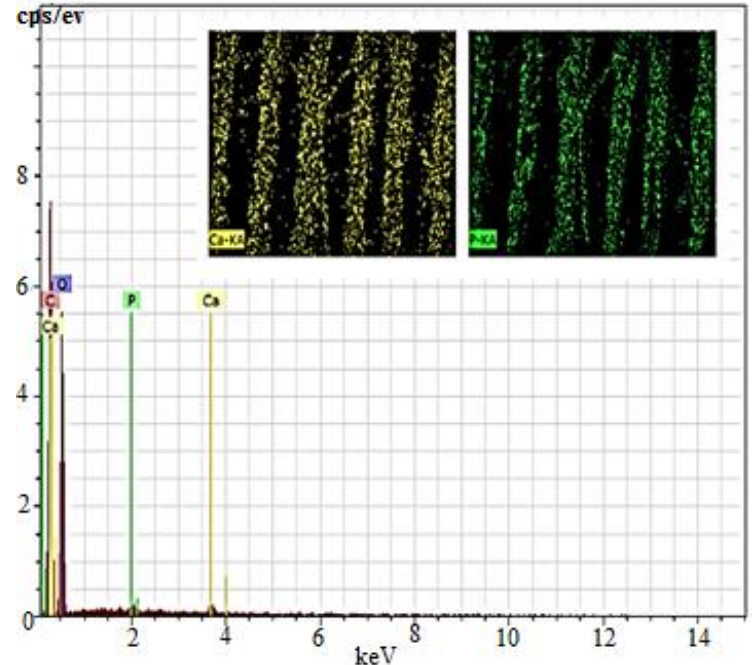

(b)

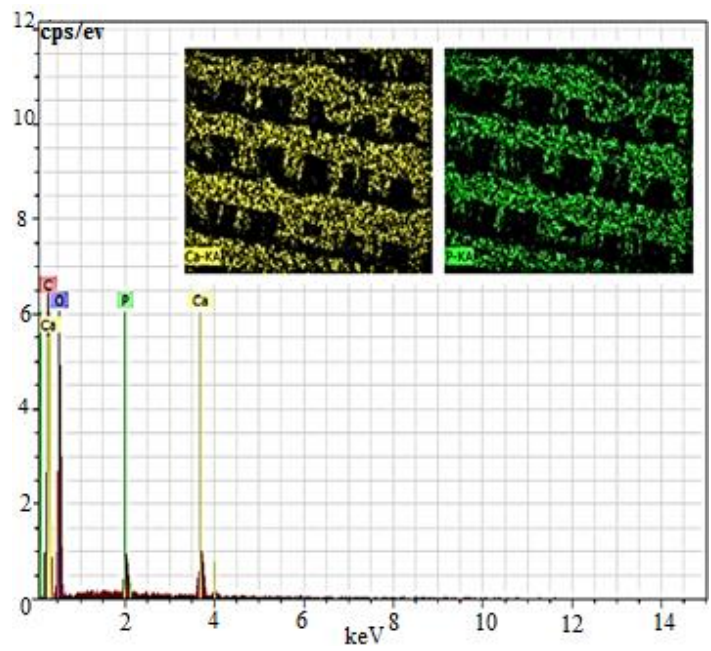

(d)

Figure 5. Spectrum and surface mapping of the scaffold in the ratios of (a) 100/0, (b) 95/5, (c) 90/10, and (d) $80 / 20$

The micro-structural 3D printing view of the SEM composite of the scaffold frameworks at various ratios is shown in Figure 6. Analysing the data shown in Figure 6, referring to the cross-sections of the scaffolds, a relevant aspect is the deposition of two subsequent layers in the same direction, with each layer being approximately $0.3 \mathrm{~mm}$ thick. Possibly, such a layer deposition feature is related to the printing parameters, such as the height of the deposited layer. The FDM machine software responsible for calculation how many layers should be deposited in the same direction so that it reached the projected height. Since the projected height of each pore was $0.7 \mathrm{~mm}$, the printer made a second deposition in the same direction to reach that height. 


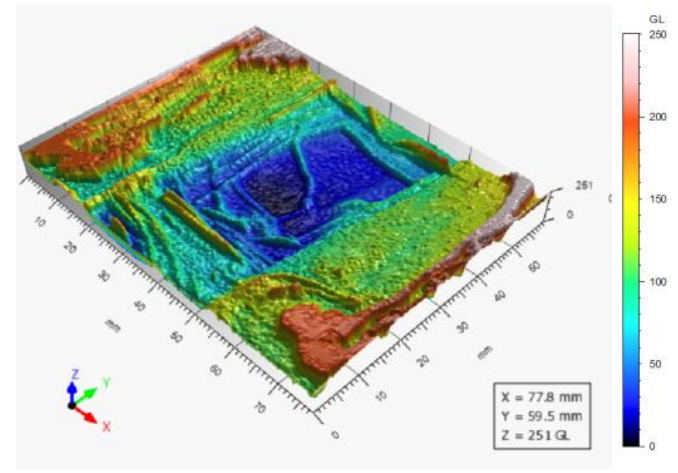

(a)

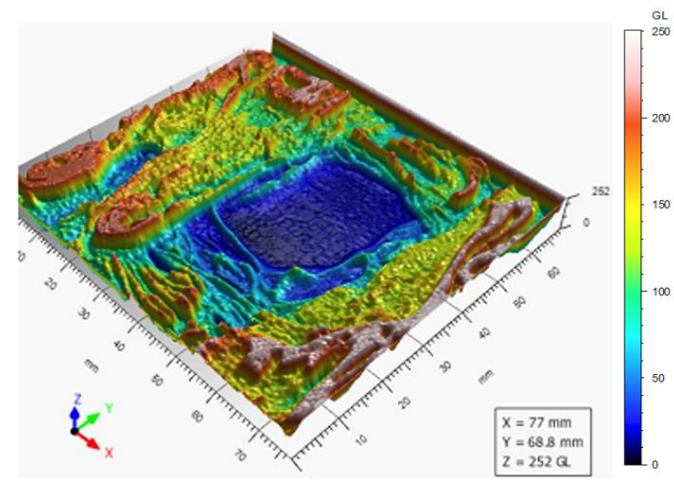

(c)

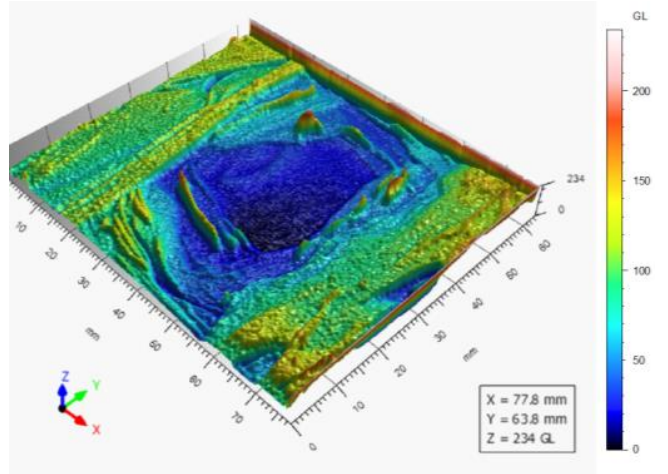

(b)

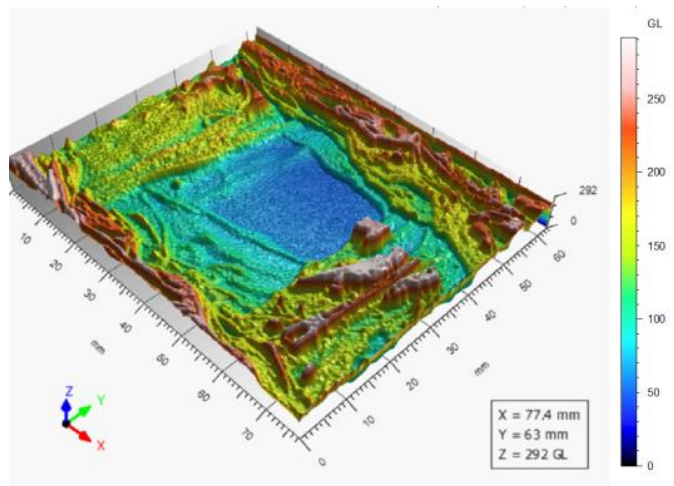

(d)

Figure 6. Micro-structural 3D printing view of SEM composite of the scaffold frameworks at (a) 100/0, (b) $95 / 5$, (c) $90 / 10$, and (d) $80 / 20$ ratios

\subsection{Mechanical compression test}

Figure 7 shows the percentage deformation, with curves relative to test bodies subjected to compressive stresses on the XY plane of the frames. The lines of the graph represent before (CP1), during (CP2) and after the test (CP3).

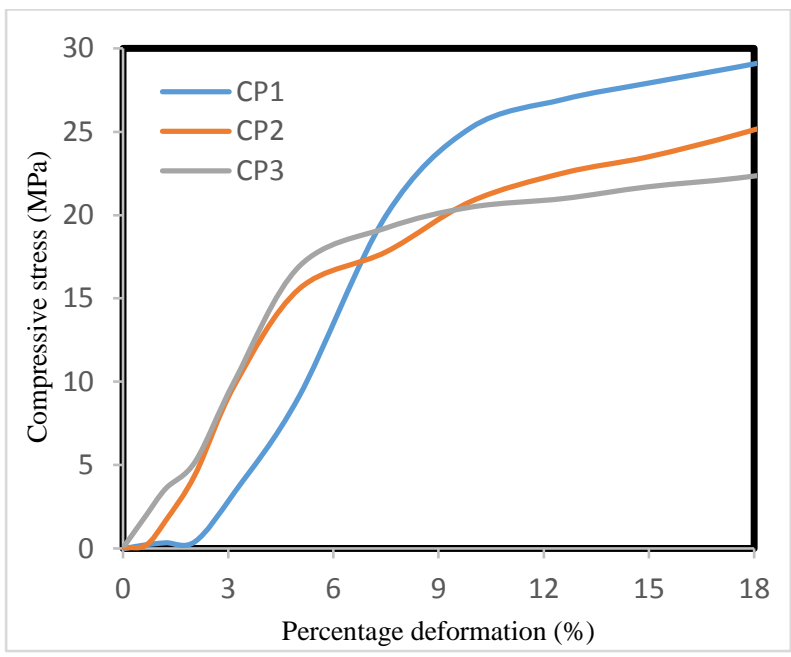

(a)

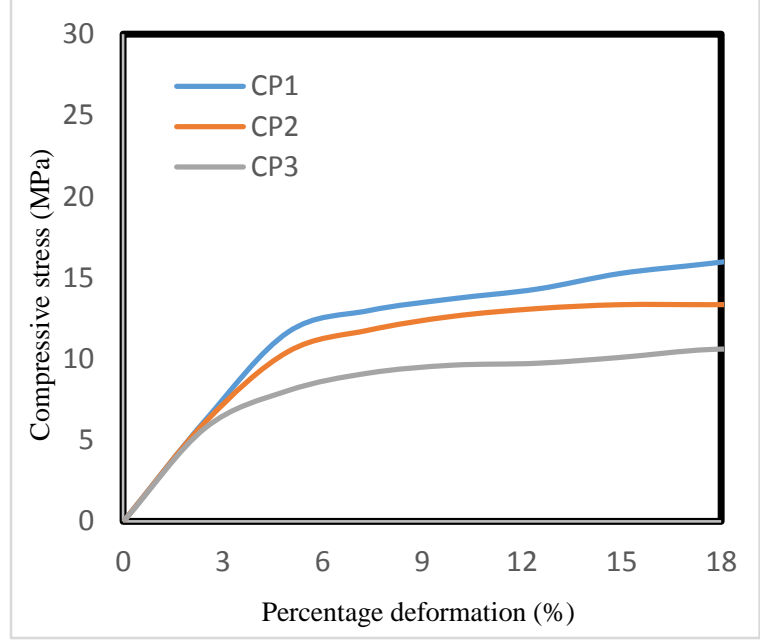

(b) 


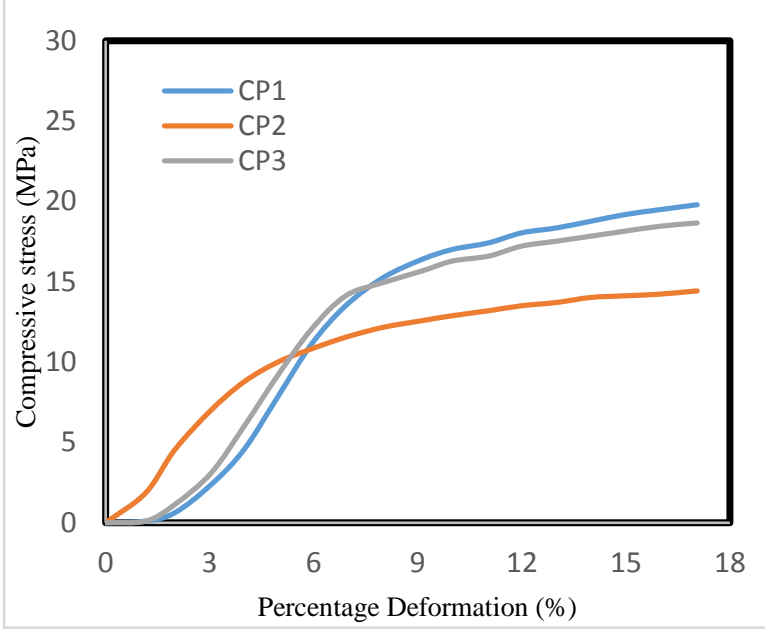

(c)

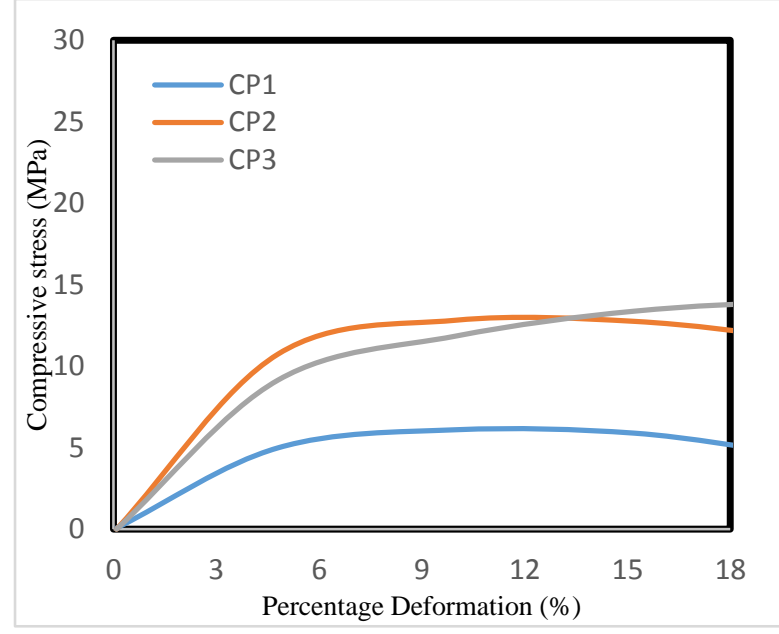

(d)

Figure 7. Compressive stress via deformation of PLA/CHA scaffolds in (a) 100/0, (b) 95/5, (c) 90/10, and (d) $80 / 20$ mass ratio

Figure 7 shows the results of general stress against deformation of a scaffold subject to a compression test in 3 distinct stages. Based on the data shown in Figure 7, it is possible to see a fall in the modulus of elasticity after the addition of cHA. However, even if there is a drop in these values with low concentrations of carbonatite, as the percentage of cHA increases, the modulus of elasticity increases, surpassing the value found in scaffolds made with pure PLA. In none of the samples were the values close to those ideal for human bones, possibly due to the poor chemical interaction between the polymer matrix and the ceramic filler.

As shown in Figure 8, the zooming factor of the microstructure was determined using a $\mathrm{x} 4$ micrometer of angular profile 180GL at spatial frequency 0.111 to $0.138 \mathrm{~mm}^{-1}$, along with the resultant amplitude, dominant spatial frequency (DSF), and maximum amplitude (MA) for the four ratios. The tolerance limit scale-sensitive fractal analysis of the $\mathrm{R}^{2}$ coefficient of the specimens was 0.915 , a good value of tolerance limit, for the $100 / 0$ ratio, 0.715 , a fragile tolerance limit, for both the $95 / 5$ and $90 / 10$ ratios, and 0.955 for the $80 / 20$ ratio.

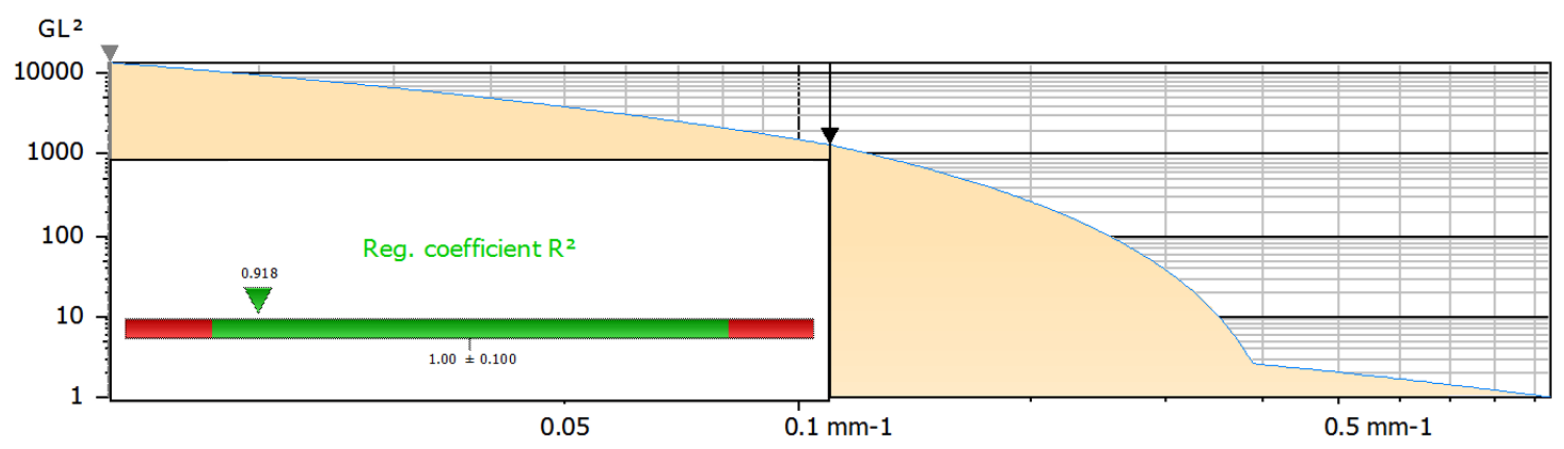

(a) 


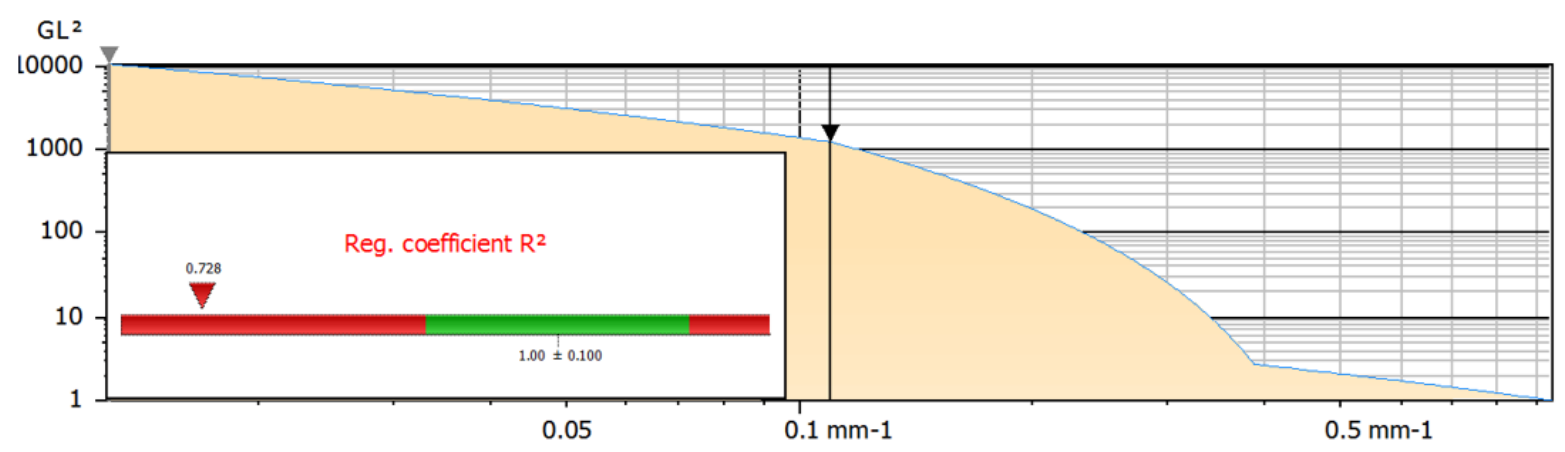

(b)

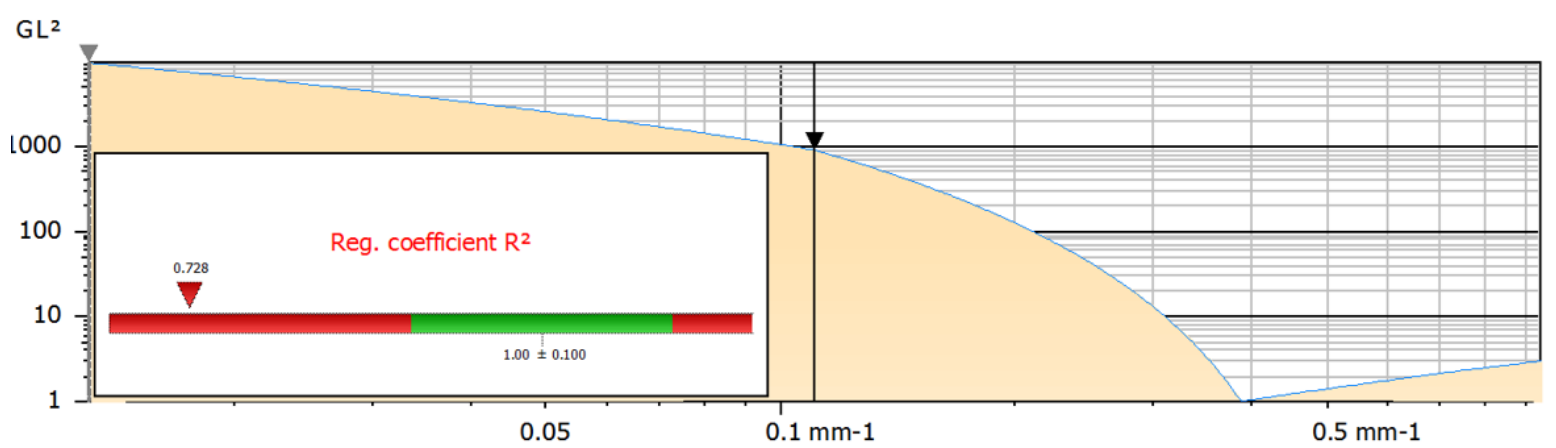

(c)

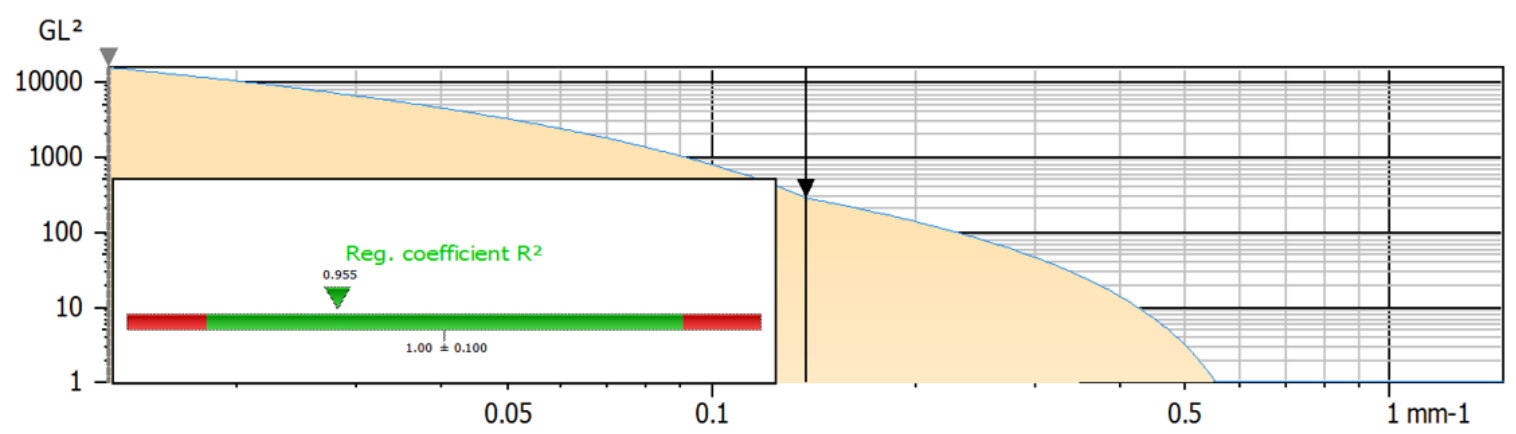

(d)

Figure 8. Averaged power spectrum density (PSD) of the 3D printing view of the bone composite in luminance converted form, (a) amplitude $1308 \mathrm{GL}^{2}$, DSF $0.0129 \mathrm{~mm}^{-1}$, MA $13724 \mathrm{GL}^{2}$, (b) amplitude $1210 \mathrm{GL}^{2}$, DSF $0.0129 \mathrm{~mm}^{-1}$, MA $10295 \mathrm{GL}^{2}$, (c) amplitude $901 \mathrm{GL}^{2}$, DSF $0.013 \mathrm{~mm}^{-1}$, MA $9595 \mathrm{GL}^{2}$, (d) amplitude $284 \mathrm{GL}^{2}$, DSF $0.0129 \mathrm{~mm}^{-1}$, MA $15532 \mathrm{GL}^{2}$

Figure 8 shows that the values for compressive strength fall with the addition of cHA. However, all samples present values within the range 2 to $12 \mathrm{MPa}$, coinciding with the expected values for trabecular bones. The frameworks produced by composite materials loaded on the XZ plane present resistance values within the same range. However, when the results of the test specimens subject to loading on the XY plane are analysed, it is possible to see an increase in the resistance in the case of the scaffolds produced with a 90/10 PLA/cHA mass ratio, possibly due to the smaller presence of failures. These low values of resistance can be justified by the second deposition in the same direction, as shown in Figure 8. This dual deposition leads to misalignment, facilitating the separation between layers, and thus damaging the mechanical characteristics of the frameworks. Double-deposited frames in the same direction present lower values of compressive strength than those printed with only one layer in each direction. 


\section{Conclusions}

One significant application of 3D printing is fabricating bone scaffolds for replacing injured tissue with biodegradable material with appropriate mechanical properties. The majority of 3D printer inks used in bone or cartilage applications have an inherent stiffness. A balance between ingredients must be struck for the creation of an appropriate printable biomaterial. In this research, a composite biomaterial, composed of a PLA matrix reinforced with carbonated HA particles in proportions of $10 / 0,95 / 5,90 / 10$ and $80 / 20$ is tested for suitability as a replacement for bone tissue. The mechanical properties observed indicate a low interaction between PLA and cHA, since, in general, the moduli of elasticity and compressive strength of the scaffolds produced in proportions 95/5, 90/10 and 80/20 have lower values than those found in the pure polymer. This weak interaction in the composite generates defects which act as stress concentrators, reducing the mechanical strength of the framework. It can be concluded from the results shown in Figure 8, that the composite 80/20 is better than the others. Another aspect that may influence the mechanical properties is the double deposition of filaments which facilitates the detachment of the layers, causing the layers of the test body to peel off earlier than expected, reducing the modulus of elasticity and strength. It is shown that an increase in the proportion of carbonatite in the frameworks can be definitive for bone regeneration. However, a more significant interaction between the matrix and the composite load is necessary, to achieve mechanical aspects close to those found in bone tissue. Further study could be done into the printing parameters, evaluating the impact of changes on mechanical behaviour and the compatibilisation between PLA and cHA. Finally, reproducibility concerns, quality control issues and regulatory hurdles need to be addressed before $3 \mathrm{D}$ printed scaffold devices can reach medical markets.

\section{Acknowledgement}

This project is funded by the Higher Education Innovation Fund (HEIF) of De Montfort University 2017-2018, UK: Research Project No. 0042.07.

\section{References}

[1] J.R. Tumbleston, D. Shirvanyants, N. Ermoshkin, R. Janusziewicz, A.R. Johnson, D. Kelly, K. Chen, R. Pinschmidt, J.P. Rolland, A. Ermoshkin, E.T. Samulski, J.M. DeSimone, "Continuous liquid interface production of 3D objects", Science, vol. 347, pp. 1349-1352, 2015.

[2] S. Aghyarian, E. Bentley, T.N. Hoang, I.M. Gindri, V. Kosmopoulos, H.K.W. Kim, D.C. Rodrigues, "In vitro and in vivo characterization of premixed PMMA-cap composite bone cements", ACS Biomater. Sci. Eng., vol. 3, p. 2267-2277, 2017.

[3] H.R. Bakhsheshi-Rad, E. Hamzah, M. Daroonparvar, R. Ebrahimi-Kahrizsangi, M. Medraj, "In-vitro corrosion inhibition mechanism of fluorine-doped hydroxyapatite and brushite coated $\mathrm{Mg}-\mathrm{Ca}$ alloys for biomedical applications," Ceram. Int, vol. 40, p. 7971-7982, 2014.

[4] C. Robo, C. Öhman-Mägi, C. Persson, "Compressive fatigue properties of commercially available standard and lowmodulus acrylic bone cements intended for vertebroplasty", Journal of the Mechanical Behavior of Biomedical Materials, vol. 82, p. 70-76, 2018. 
[5] F. Pahlevanzadeh, H.R. Bakhsheshi-Rad, E. Hamzahc, "In-vitro biocompatibility, bioactivity, and mechanical strength of PMMA-PCL polymer containing fluorapatite and graphene oxide bone cements", Journal of the Mechanical Behavior of Biomedical Materials, vol. 82, p. 257-267, 2018.

[6] H.N. Chia, B.M. Wu., "Recent advances in 3D printing of biomaterials," J. Biol. Eng., vol. 9, no. 4, pp. 2-14, 2015.

[7] H. Cheng, C. Jin, J. Wu, S. Zhu, Y.J. Liu, J. Chen, "Guards at the gate: physiological and pathological roles of tissue-resident innate lymphoid cells in the lung", Protein Cell, vol. 8, p. 878-895, 2017.

[8] B.I. Oladapo, S.A. Zahedi, F. Vahidnia, O.M. Ikumapayi, M.U. Farooq, "Threedimensional finite element analysis of a porcelain crowned tooth", Beni-Suef University Journal of Basic and Applied Sciences, 2018, https://doi.org/10.1016/j.bjbas.2018.04.002

[9] H.J Kim, U. Kim, H.S. Kim, C. Li, M. Wada, G.G. Leisk, D.L. Kaplan, "Bone tissue engineering with premineralized silk scaffolds", Bone, no. 42, p. 1226-1234, 2008

[10] B.I. Oladapoa, S.A. Zahedi, S.C. Chaluvadi, S.S. Bollapalli, M. Ismail, "Model design of a superconducting quantum interference device of magnetic field sensors for magnetocardiography", Biomedical Signal Processing and Control, vol.46, p.116-120, 2018

[11] A.M. Adeoye, J.F. Kayode, B.I. Oladapo, S.O. Afolabi, "Experimental analysis and optimization of synthesized magnetic nanoparticles coated with PMAMPC-MNPs for bioengineering application", St. Petersburg Polytech. Univ. J. 3 (4) p.333-338, 2017

[12] S.A. Zahedi, M. Demiral, A. Roy, V.V. Silberschmidt, "FE/SPH modelling of orthogonal micro-machining of fcc single crystal", Computational materials science, vol. 78, p.104-109, 2013

[13] S.A. Zahedi, A. Roy, V.V. Silberschmidt, "Modeling of micro-machining single-crystal fcc metals", Procedia CIRP, vol. 8, p.346-350, 2013

[14] B.I. Oladapo, A.M. Adeoye, M. Ismail, "Analytical optimization of a nanoparticle of microstructural fused deposition of resins for additive manufacturing", Composites Part B, vol.150, p.248-254, 2018

[15] M. Silva, R. Felismina, A. Mateus, P. Parreira, C. Malça, "Application of a hybrid additive manufacturing methodology to produce a metal/polymer customized dental implant", Procedia Manufacturing, vol. 12, p. 150 - 155, 2017.

[16] S. Patra, V. Young, "A review of 3D printing techniques and the future in biofabrication of bioprinted tissue", Cell Biochem. Biophys, vol. 74, p. 93-98, 2016.

[17] R.C. Dutta, M. Dey, A.K. Dutta, B. Basu, "Competent processing techniques for scaffolds in tissue engineering," Biotechnology Advances, no. 35, p. 240-250, 2017.

[18] S. Ahmed, Annu, A. Ali, J. Sheikh, "A review on chitosan centred scaffolds and their applications in tissue engineering," Int. Jou. of Bio. Macro, vol. 116, pp. 849-862, 2018.

[19] S. Bhattacharyya, J. Varga, "Endogenous ligands of TLR4 promote unresolving tissue fibrosis: implications for systemic sclerosis and its targeted therapy", Immunol. Lett, vol. 195, p. 9-17, 2018. 
[20] S.J. Cai, C.W. Li, D. Weihs, G.J. Wang, "Control of cell proliferation by a porous chitosan scaffold with multiple releasing capabilities Sci. Technol," Adv. Mater., vol. 18, pp. 987-996, 2017.

[21] V. Raeisdasteh Hokmabad, S. Davaran, A. Ramazani, R. Salehi, "Design and fabrication of porous biodegradable scaffolds: a strategy for tissue engineering", J. Biomater. Sci. Polym. Ed., vol. 28, pp. 1797-1825, 2017.

[22] C. Wu, W. Fan, Y. Zhou, Y. Luo, M. Gelinsky, J. Chang, Y. Xiao, "3D-printing of highly uniformCaSiO3 ceramic scaffolds: preparation, characterization and in vivo osteogenesis", J. Mater. Chem, vol. 22, no. 24, p. 12288-12295, 2012.

[23] S. Naahidi, M. Jafari, M. Logan, Y. Wang, Y. Yuan, H. Bae, B. Dixon, P. Chen, "Biocompatibility of hydrogel-based scaffolds for tissue engineering applications", Biotechnology Advances, no. 35, p. 530-544, 2017.

[24] Y. Liu, H. Wang, N. Li, X. Zhou, W. Gao, J. Jiao, "A novel $\beta$-calcium sulphate hemihydrate injectable biocement: in vitro and in vivo study", Sci. Adv. Mater., vol. 7 , no. 12, p. 2603-2610, 2015.

[25] Z. Ruana, D. Yao, Q. Xu, L. Liu, Z. Tian, Y. Zhua., "Effects of mesoporous bioglass on physicochemical and biological properties of calcium sulfate bone cements", Applied Materials Today, vol. 9, p. 612-621, 2017.

[26] D.T. Yang, D. Zhang, D. Dwayne, D. Arola, "Fatigue of the bone/cement interface and loosening of total joint replacements", International Journal of Fatigue, vol. 32, pp. 1639-1649., 2010.

[27] Z. Li, H.R. Ramay, K.D. Hauch, D. Xiao, M. Zhang, "Chitosan-alginate hybrid scaffolds for bone tissue engineering Biomaterials", biomaterials, vol. 26, pp. 39193928, 2005.

[28] S. Li, S.A. Zahedi, V. Silberschmidt (2017) Numerical simulation of bone cutting: Hybrid SPH-FE approach, Numerical methods and advanced simulation in biomechanics and biological processes, 187-201, Book chapter

[29] A.V. Zaryanov, D.K. Park, J.G. Khalil, K.C. Baker, J.S. Fischgrund, "Cement augmentation in vertebral burst fractures", Neurosurg. Focus, vol. 37, no. 1, p. 2014, E5.

[30] A.B. Yeatts, J.P. Fisher, "Bone tissue engineering bioreactors: Dynamic culture and the influence of shear stress", Bone, no. 48, p. 171-181, 2011.

[31] I. Ajaxon, C. Persson, "Compressive fatigue properties of a commercially available acrylic bone cement for vertebroplasty", Biomech. Model. Mechanobiol, vol. 13, p. 1199-1207, 2014. 